\title{
WHY AND HOW CULTURE MATTERS IN COMMUNITY INTERORGANIZATIONAL STRUCTURE
}

\author{
MOSES A. BOUDOURIDES
}

\begin{abstract}
Our aim here is to plead for the significance of cultural considerations of overlapping inter-attitudinal patterns right next to well established structural considerations of interorganizational networks based on overlapping membership patterns. In particular, we examine how the analytic methodological incorporation of cultural attributes or attitudes might enhance our understanding of structural community categorizations in interorganizational networks. For this purpose, we analyze data of the International Peace Protest Survey (IPPS) on the world-wide peace protests of February, 15, 2003, in order to manifest the added value offered by the consideration of the culturestructure duality in participation studies.
\end{abstract}

\section{Structure, Culture and Social Networks}

In conformity to the "cultural turn" in modern sociology, culture and structure (or the cultural and the social) are usually conceived intermingled in a complex duality (Friedland \& Mohr, 2004). As a matter of fact, that was also the fundamental conviction of the structural framework in the general sociology of Harrison C. White (2008a), which, in particular, was preceded by his less known "catnets" thesis aiming to provide a preliminary analytic formalization of the structure-culture duality (White, 2008b). Similar sources of inspiration were met in the dogma of "relational sociology" elaborated in the famous Manifesto for a Relational Sociology of Mustafa Emirbayer (1997) and in the much broader discussion on the relationship between structure and agency that took place in the context of collective action (Emirbayer \& Mische, 1998, Emirbayer \& Goodwin, 1994) - although the latter was overtly critical to White's approach. In any case, under the connotation of all the fundamental contributions on structural-cultural relationalism - and beyond their apparent disparities - the term "cultural turn in social network analysis" (Mützel, 2009) is sometimes used in the context of certain analytic approaches, which prefer to treat action-based social networks as both constituting and constituted by culture.

Now, let us recall that the inaugurating idea of social network analysis was that social structure is not to be understood in terms of categorical attributes and, in particular, of any cultural identifications of individual or collective actors, but solely

Date: July 9, 2011.

Part of this work was done while the author was visiting the Eindhoven University of Technology in the Netherlands from October 2010 to January 2011, the Antwerp Management School in Belgium during February and March 2011 and the Université Paris Dauphine in France in April 2011. It is intended to be a first draft of the author's contribution to a paper to be written with Iosif Botetzagias and Stefaan Walgrave. 
on the basis of social interaction among actors. Nevertheless, this anticategorical imperative of network analysis (Emirbayer \& Goodwin, 1994) should not be construed as if the relational theorization of structure and culture was postulating that actors, ties and attributes were all independent to each other. On the opposite, it is in the enmeshing of structure and culture that meaning arises and identities emerge concurrently and viscerally coupled with materialized patterns of structural rootedness, i.e., embeddedness. For instance, from Harrison C. White's (2008a) point of view, actors make sense of themselves and the world they live, while striving to establish control over surrounding ambiguities and contingencies and to settle their social footing with other actors or entities in their everyday life.

In particular, when structure is formally represented by a pattern of relations (or ties) among actors and culture is schematically reflected into a multiplicity of variable and diverse attributes or attitudes that actors exhibit, there are two coupled mechanisms and processes of social change and morphogenesis, which are commonly examined in social network analysis. On the one hand, it is through the mechanism of network influence that structure may determine culture, in the sense that actors' attitudes might change due to the influence exerted from their network localities. At the same time, on the other hand, it is through the mechanism of network selection that culture may determine structure, in the sense that culture may motivate actors to establish new connections or severe older ones; in this way, actors manage to interlace their structural entanglements, while driven by the cultural attributes they possess as holders of attitudes conforming to norms or codes of behavior, which are dictated (positively or negatively) by the prevailing cultural dispositions (habitus, preferences, morals etc.). However, these two mechanisms and the processes they set off are not mutually exclusive, but, rather, intertwined, as many network theorists have already argued (Erickson, 1988, Leenders, 1997). Thus, for instance, in the cases of homophily (Lazarsfeld \& Merton, 1964, McPherson et al. , 2001) or focus constraint (Feld, 2000), cultural attributes and actors' attitudes were studied as predictors of structure (e.g., Robins et al. , 2001b, 2001a). Furthermore, in other formal studies, structural ties were taken as predictors of cultural attributes and actors' attitudes (cf., Robins et al. , 2001a; relevant statistical approaches for both cases are discussed by Robins et al. , 2007, Steglich et al. , 2007).

\section{Interorganizational Networks and Culture}

In social network analysis, interorganizational networks are understood as networks, which are formed by a particular class of collective actors, organizations. In such networks, ties among actors might be of two types. One is the type of direct interorganizational ties - such as, for instance, on the macro-level, various exchanges among organizations or, on the micro-level, some sort of formal or informal interactions among members of organizations. Analytically, these networks are commonly described as one-mode networks, since they involve a single mode of actors (organizations) together with the pattern of ties among them. However, here, we will be concerned with the other type of indirect (or "latent" in the terminology of Alberto Melucci, 1989, 1996) ties among organizations, which are commonly postulated to exist through and because of the occurrence of patterns of multiple-memberships 
of individual actors, who simultaneously participate or are involved in more than one of the studied organizations. From the analytic (graph-theoretic) point of view, such structures are called two-mode networks (or bipartite graphs), as they involve two modes of actors, the mode of individuals and the mode of organizations, together with the pattern of associations (affiliations or memberships) among individuals and organizations - notwithstanding, without assigning any direct linkages among either one of the actors inside each mode.

In fact, this is a particular instance of the renowned "duality" (in this case) between individuals and organizations (Simmel, 1955, Breiger, 1974). Moreover, here, we will be concerned only with the particular case of interorganizational networks, which are formed by patterns of participation of individuals (activists) in various (social movement) political organizations. In this setting, by being members of (or affiliated to) different social movement organizations, activists create and sustain the linkages of the corresponding interorganizational networks (Diani, 2004), through which personal identities of activists and collective identities of social movements may be structurally constituted and, at the same, these identities may be culturally weaving the complex entanglements of activists and social movements. For instance, such processes were activated by those that Hedström et al. , 2000 , have called "socialist agitators," i.e., traveling activists operating as human bridges between movement milieus in different localities.

When it comes to the coupling of structure and culture in interorganizational networks, there has not yet been attained any considerable methodological or formal advancement for the case of two-mode networks similar to the analytical and statistical studies on homophily and focus constraint applied to the case of onemode networks (that were cited above). However, it is our aim to discuss here some first steps on a graph-theoretic formalization of how culture matters in the analysis of certain structural aspects of interorganizational networks (related to community categorization, as we will explain in the next section).

To do so, we need first to set out with an elementary notation and certain distinctive terminology of some rather trivial concepts in interorganizational networks that we will be using here - as a matter of fact, we are going to use almost the same notation with Ronald Breiger (1974). So, let us assume that we have collected a set of empirical data through a questionnaire (or interviews etc.), in which a number of activists - sometimes referred as participants - report their participation (or involvement or affiliation or membership etc.) to a number of (social movement) organizations. In particular, we denote by $G$ the set of all organizations included in the data and by $P$ the set of all activists, who are distributed (while overlapping) among these organizations. If for any set $X,|X|$ denotes the cardinality of $X$, i.e., the count of members of $X$, we assume that $|G|=g$ meaning that $G$ is composed of $g$ organizations and $|P|=p$, i.e., $P$ is composed of $p$ activists (social movement participants). Hence, such data correspond to a matrix $A=A(P, G)$ of order $p \times g$ (i.e., a matrix composed of $p$ rows and $g$ columns), which may be represented by a two-mode network (or a bipartite graph) of activists and organizations, denoted as $\mathcal{H}=\mathcal{H}(P, G)$. 
Using the generic symbols $O_{i}, O_{j}, O_{k}, \ldots$ for organizations, given two arbitrary organizations, $O_{i}$ and $O_{j}$, let us denote by $B_{i j}$ the set of all participants, who are affiliated to both organizations $O_{i}$ and $O_{j}$. Note that $B_{i j}$ is a subset of $P$ (formally, one writes $B_{i j} \in 2^{P}$, the power set of $P$ ) and, denoting by $\left|B_{i j}\right|$ the cardinality of these (sub)sets, then $\sum_{i, j \in P}\left|B_{i j}\right| \geq p=|P|$, exactly because members (participants) are overlapping among organizations. From now on, we will be referring to the set $B_{i j}$ as the "band of overlapping members" in the two organizations $O_{i}$ and $O_{j}$. Of course, the name makes sense only as far as the set is non-empty, $B_{i j} \neq \varnothing$, i.e., as far as there exists at least one activist involved with both organizations. If we find all possible (non-empty) bands of membership overlaps throughout our data, then the fundamental postulate of the persons-groups duality theory says that this set may assess the existence of indirect relationships (ties) among organizations. The idea of how this is done is the following:

- If, for two organizations $O_{i}$ and $O_{j}$, it happens that $B_{i j}=\varnothing$ (i.e., if there exist no overlapping members to both organizations), then the organizations $O_{i}$ and $O_{j}$ are not linked to each other. Thus, the necessary condition for two organizations to be linked together in the interorganizational network is that the corresponding (to these organizations) band of overlapping members is a nonempty set.

- If the cardinality (i.e., the count of membership) of a band $B_{i j}$ of overlapping members to organizations $O_{i}$ and $O_{j}$ is larger than the cardinality of a band $B_{k l}$ of overlapping members to two other organizations $O_{k}$ and $O_{l}$, then the former organizations $\left(O_{i}\right.$ and $\left.O_{j}\right)$ possess an intenser tie than the latter $\left(O_{k}\right.$ and $\left.O_{l}\right)$.

Therefore, as far as one admits that the totality of bands of membership overlaps may assess the existence of all possible interorganizational ties and, furthermore, that these overlaps may measure the intensity of the corresponding ties, then one obtains the projection of the two-mode network $\mathcal{H}=\mathcal{H}(P, G)$ of activists and organizations on a one-mode network $\mathcal{G}=\mathcal{G}(G)$ of organizations, which is the interorganizational network we intend to study. Actually, $\mathcal{G}$ is a weighted network, meaning that its links possess a weight, which is expressed by the numerical cardinality of the occurring bands of overlapping membership. For instance, the weight of the (indirect) link among organizations $O_{i}$ and $O_{j}$ is equal to $\left|B_{i j}\right|$. Now, noting that (for $i=j$ ) $\left|B_{i i}\right|$ is just the (total) membership of organization $O_{i}$, it turns out that the normalized weight $\frac{\left|B_{i j}\right|}{\min \left\{\left|B_{i i}\right|,\left|B_{j j}\right|\right\}}$ may give rise to an indicator of organizational structural embeddedness, according to Gulati \& Gargiulo (1999) (cf., Andretta et al. , 2009). In any case, neglecting the aforementioned normalization, the adjacency matrix of the one-mode interorganizational network $\mathcal{G}$ is a symmetric matrix $A=A(G)$ of order $g \times g$, where the $(i, j)$ entry of $A$ is equal to the weight $\left|B_{i j}\right|$. Formally, ignoring diagonal terms, this (adjacency) matrix is given by the following matrix product (Breiger, 1974):

$$
A(G)=A(P, G)^{T} A(P, G),
$$

where $A(P, G)^{T}$ is the transpose matrix of the two-mode matrix $A(P, G)$ - typically, extracted from the data of the questionnaire.

At this point, let us make a rather evident remark on how an interorganizational network differs from a personal network (say, a network among persons being tied 
by such relationships as friendship, support, affect etc.). In a personal network ties are sustained by a certain direct action that an actor (person) may develop with regards to another actor (person). However, in an interorganizational network, collective actors (organizations) are not seen as directly related to each other, but only indirectly through the intermediation of individual actors (participants to organizations). In other words, a tie between two organizations is only sustained indirectly by those participants who act as a kind of duplex proxies (or brokers) of these organizations. In our terminology, these are the participants who constitute the discrete bands of overlapping membership and, in this oblique way, they may bind organizations together. Of course, such an indirect linkage of organizations by intermediate bands of membership overlaps is only speculated to represent and surrogate the actual (one-modal) interorganizational (social) relationships.

Now, a direct way to see the traces of culture on interorganizational networks would be by analyzing how overlaps in participants' membership are differentiated in the presence of cultural attributes or attitudes that actors (participants) possess. The underlining idea has been already explored by Cornwell \& Harrison (2004), who, in their study of the interorganizational network among unions and voluntary associations, have argued that: "Analyzing how members of various sociodemographic groups tie unions to other organizations may provide additional insight into the basis of unions' embeddedness" (p. 869). For this purpose, in order to analyze the influence of certain sociodemographic attributes (such as sex, race, class and religion) on the way unions are embedded in a broader organizational ecology, Benjamin Cornwell and Jill Ann Harrison first isolated the patterns of membership overlaps within each attribute and then they tested which attributes were more effective in tying unions into the overall interorganizational network.

Thus, a graph-theoretic formulation of how to analyze the effect of cultural attributes on an interorganizational network structure might proceed as follows. (Of course, this is not the only way to analyze such multi-partite network data; for an alternative tripartite structural analysis, see Fararo \& Doreian, 1984.) Let $\mathcal{Q}$ be a (finite) set of attributes or attitudes possessed by activists (participants) in $P$ reporting their affiliation to multiple (social movement) organizations in $\mathcal{G}$, the way this information was encoded in the two-mode data matrix $A$ extracted from an empirical survey (questionnaire etc.). Obviously, each activist (participant) might declare possession of multiple attributes or attitudes from $\mathcal{Q}$. In any case, for each attribute $\alpha \in \mathcal{Q}$, let us denote by $B_{i j}^{(\alpha)}$ the set of all participants, who are simultaneously affiliated to two organizations $O_{i}$ and $O_{j}$, while, at the same time, they are both possessing the attribute $\alpha$. (Practically speaking, to say that a participant "possesses an attribute or attitude" means that this participant has responded affirmatively to the question of the questionnaire that corresponds to this attribute or attitude.) Similarly to the name we have given for the set $B_{i j}$, let's call $B_{i j}^{(\alpha)}$ "band of overlapping members possessing the attribute or attitude $\alpha$ " or, shortly, just "band of overlapping $\alpha$-members." Trivially, $B_{i j}^{(\alpha)}$ is a subset of $P^{(\alpha)}$, i.e., of the set of all participants possessing attribute $\alpha$, and $\sum_{\alpha \in \mathcal{Q}}\left|B_{i j}^{(\alpha)}\right| \geq\left|B_{i j}\right|$, exactly because members (participants) may overlap among attributes. Therefore, similarly as before, one obtains the projection of any one of the two-mode networks 
$\mathcal{H}^{(\alpha)}=\mathcal{H}^{(\alpha)}\left(P^{(\alpha)}, G\right)$ of $\alpha$-activists and organizations on the one-mode interorganizational and inter-attributional networks $\mathcal{G}^{(\alpha)}=\mathcal{G}^{(\alpha)}(G)$. Clearly, the $\mathcal{G}^{(\alpha)}$ 's are again represented by weighted graphs with symmetric adjacency matrices equal to the product $\left\{A^{(\alpha)}\right\}^{T} A^{(\alpha)}$, where $A^{(\alpha)}$ is the two-mode data matrix restricted just to $\alpha$-participants.

Then, in order to elucidate the idea that culture may influence structure in interorganizational networks, it is very instructive to adopt a metaphorical scheme of immersing ourselves into a rather vivid perspective. In such a pictorial account, cultural attributes and actors' attitudes may be graphically conceived as distinct 'colors,' which are intertwining a 'colorful' patchwork of various partitions of bands of overlapping membership that constitute an interorganizational network. However, when one focuses exclusively on a certain 'color' in these partitions, i.e., when bands of membership overlaps are restricted only on actors (participants) satisfying a single cultural attribute or attitude, then the structure of the interorganizational network might appear different than in patterns, in which 'colors' were either erased or superimposed. Hence, from a graphical point of view, to decipher the volatile traces of culture amounts to be able to discern the added value that a cultural 'coloring' might give to the 'colorless' bulk of an interorganizational network, in which cultural attributes and actors' attitudes were ignored or compensated. Needless to say that, since bands of co-participants are typically overlapping (in the sense that, typically, there exist participants affiliated to multiple organizations, where 'multiple' means larger than two), the decomposition of the overall interorganizational network into its 'colored' components is not additive or linear. Therefore, it is possible that certain structural characteristics may become more visible, if not revealed more clearly, only when an interorganizational network is 'colored' by a certain attribute or attitude, while some other structural features might be dumped, when the network appears deprived of the cultural qualities of actors. Having said that, we should not forget that, since an interorganizational network is represented by a 'weighted' graph, the overall effect of culture on structure depends on how the existing cultural attributes and attitudes of actors are distributed over the interorganizational network ties. As a consequence, the cultural effect on structure is more salient for certain structural characteristics of the interorganizational network than others. In the next section, we are going to discuss an example of a structural feature, which is not only quite sensitive to cultural variation, but, moreover, it recapitulates substantively the celebrated duality between structure and culture.

\section{Communities and Culture}

We claim that an appropriate structural feature, on which cultural effects may plainly imprint, is that which is usually named "community structure." The defining idea is very simple: in a social network, a community partitioning (or community structure) consists of distinct communities, which are identified with cohesive groups of tight-knit actors (Wasserman \& Faust, 1994, p. 249). In other words, actors inside a community should be relatively more densely connected to each other than how (relatively sparsely) actors among different communities were connected. 
Before entering into a technical discussion about the mathematical and computational operationalization of a community partition in a social networks and, in the sequel, about the cultural effect on interorganizational network structures, it is very timely to examine what the concept of community really signifies in social theory. Undoubtedly, to say to which community one belongs is a peculiar type of a categorization. It is rather a group or collective categorization (a social categorization, on a more comprehensive level). In fact, a community categorization has nothing to do with what a secluded individual says or imagines of oneself or even of one's group peers. To categorize the community to which one belongs is not an issue of an enunciated self-identification, even when an individual speculates on existing affinities with other individuals. For a community does not consist of a mere aggregation of persons, conceived as self-reliant subjects, who cogitate about their destiny or recognize themselves in arbitrary or sempiternal terms outside the reality of their social worlds, their everyday practices and life. On the contrary, to categorize one's community is an issue of what one does in practical contexts, the distinctive ways one acts in mundane situations, how one interacts with or with regards to others. Even a person's autonomy (Castoriadis, 1998) or a personal identity, i.e., whatever distinguishes a person from others, are singular characteristics, which develop through a bundle of inter-personal shared significations, embedded in the relational footing of the multiplex ties, structural and cultural, that persons sustain with others in the settings of their common social spaces. Not only "communities can be seen as built out of identities rather than persons," as Harrison White (2008a, p.157) has argued, following the historical investigations of Allan Silver $(1989,1990)$, but, in addition, "the relationship between community as a complex of social relationships and community as a complex of ideas and sentiments" needs further exploration, as Craig Calhoun (2009, p. 110) was calling forth already some time ago.

Although a complete analytical exploration of this relationship is still an unsolved problem, from a formal methodological point of view, it is also challenging to try to track down a graph-theoretic approach, in which structural communities would be conceived formally as group categorizations. Actually, this is what we are trying to do here. However, in this perspective, communities should not be perceived immobilized and framed within rigid absolute boundaries. Of course, typically, there exist relational boundaries, structural cracks or interactional clefts among communities of a network partition. But these relational boundaries may interact with other existing symbolic or social boundaries inside the social spaces where actors live (Lamont \& Molnár, 2002). And the emerging outcome of the structure-culture coupling is a process of superlative complexity, like a process of social annealing with unanticipated and contingent consequences. The effect of dipping structure inside the contested or troubled waters of an encompassing fluid cultural milieu might resemble a sort of an inadvertent refraction or a mutated recombination of the preceding ordered pattern of structural categorizations. Because culture is grounded on so many contrasting or conforming distinctions, contested values or tastes or opinions, symbolic or linguistic significations, institutional or pilot arrangements, consensual or dissenting decisions or positions, social or economical inequalities or opportunities, just or unequal distribution of resources etc. 
After the previous speculative remarks, let us give a brief description of a formalmathematical approach aiming to detect the community structure of a social network. We will follow a technique based on the maximization of a certain benefit function called modularity. We have chosen this method, because it is conceptually simple and, for small graphs, one could even detect communities by a hands-on computation (e.g., for graphs with less than 5 or 6 vertices), although there are many other methods for the graph-theoretic community detection (cf., Fortunato, 2010). Just to mention two other, purely sociological, approaches for community (otherwise called cohesive or social groups) analysis, we may refer to the works of Frank (1995) and Moody \& White (2003).

To start with a short presentation of the modularity maximization technique of community detection, let $\mathcal{G}$ be a social network, which is represented, in general, by a weighted (undirected simple) graph on a set of vertices $V$ and let $n$ be the total number of vertices and $m$ the total number of edges of this graph (counting multiplicity, since the graph is weighted). Furthermore, let us denote by $A=\left\{A_{i j}\right\}$ the adjacency matrix of the weighted graph (which means that $A$ is a symmetric matrix of order $n \times n$ with its entries being non-negative integers such that $\sum_{i, j=1}^{n} A_{i j}=2 m$ ). (Of course, in the previous notation, $\mathcal{G}$ might be anyone of the projected one-mode interorganizational networks $\mathcal{G}(G)$ or $\mathcal{G}^{\alpha}(G)$, for any attribute or attitude $\alpha$ of actors in $P$.) Furthermore, let $\mathbf{C}=\mathbf{C}(\mathcal{G})=\left\{C_{1}, C_{2}, \ldots, C_{c}\right\}$ be a partition of the set of vertices $V$ of the graph (representing) $\mathcal{G}$ into $c$ subsets $C_{k}$. (Technically, such a family of subsets of $V$ forms a "partition," whenever $\bigcup_{k=1}^{c} C_{k}=V$ and $C_{k_{1}} \cap C_{k_{2}}=\varnothing$, for any $k_{1} \neq k_{2}$ in $\{1,2, \ldots, c\}$.) Then a partition $\mathbf{C}$ is called community structure of $\mathcal{G}$ and each $C_{k}$ is called a community, if a certain benefit function is maximized over this partition $\mathrm{C}$. The most commonly employed benefit function in community partitioning is the following function $Q$, called modularity, which was defined by Newman \& Girvan (2004) as:

$$
\begin{aligned}
Q= & \text { (fraction of links within communities) } \\
& -(\text { expected fraction of such links). }
\end{aligned}
$$

In the so-called null model, the above expected fraction is calculated on the basis of a hypothetical existence of a random graph, which would preserve the same degree distribution with the examined graph $\mathcal{G}$. Hence, the exact expression of modularity becomes (Fortunato, 2010):

$$
Q=\sum_{k=1}^{c}\left[\frac{l_{k}}{m}-\left(\frac{d_{k}}{2 m}\right)^{2}\right],
$$

where $l_{k}$ is the total number of edges inside community $C_{k}$ and $d_{k}$ is the sum of degrees of all vertices in $C_{k}$ (in both cases, counting multiplicity of edges, since the graph was assumed weighted). Thus, to obtain the community partitioning one has just to search for that partition, which maximizes the above modularity function $Q$. Note that this optimization problem has been proven to be NP-complete (Brandes et al. , 2008) and, therefore, only approximate optimization techniques, such as greedy algorithms, simulated annealing, extremal optimization, expectation maximization, spectral methods etc. can be practically useful.

Returning to our formal analysis of interorganizational networks, when actors (participants) may exhibit a number of cultural attributes or attitudes, next to the 
community partition $\mathbf{C}=\mathbf{C}(\mathcal{G})=\left\{C_{1}, C_{2}, \ldots, C_{c}\right\}$ of the "colorless" interorganizational network $\mathcal{G}=\mathcal{G}(G)$ (which is the one-mode projection of the two-mode network $\mathcal{H}=\mathcal{H}(P, G)$ of activists and organizations), we have a number of "colored" interorganizational networks too, arising from the supplementary information that we might have in our data (questionnaire etc.), according to which each activist (participant) may possess also a number of cultural-"colored" attributes or attitudes. Let us denote by $\mathcal{Q}=\left\{\alpha_{1}, \alpha_{2}, \ldots, \alpha_{q}\right\}$ the family of all cultural attributes or attitudes ( $q$ in number) that actors (participants) may possess in the data. This means that, corresponding to each attribute or attitude $\alpha_{l} \in \mathcal{Q}$, we have a "colored" interorganizational network $\mathcal{G}^{\alpha_{l}}=\mathcal{G}^{\alpha_{l}}(G)$ (as the one-mode projection of the two-mode network $\mathcal{H}^{\alpha_{l}}=\mathcal{H}^{\alpha_{l}}\left(P^{\alpha_{i}}, G\right)$ of $\alpha_{l}$-activists and organizations, which is extracted from the original data). Moreover, corresponding to each one of these "colored" interorganizational networks, we may find a community partition $\mathbf{C}^{\alpha_{l}}=\mathbf{C}^{\alpha_{l}}\left(\mathcal{G}^{\alpha_{l}}\right)=\left\{C_{1}^{\alpha_{l}}, C_{2}^{\alpha_{l}}, \ldots, C_{c(l)}^{\alpha_{l}}\right\}$. Note that the cardinality $c(l)=\left|\mathbf{C}^{\alpha_{l}}\right|$ of any "colored" community partition depends on the attribute or attitude $\alpha_{l}$.

Needless to say that the constituency of any "colored" community $C_{k}^{\alpha_{i}}$ depends on the attribute or attitude $\alpha_{l}$ too. Therefore, and this is very important, "colored" communities may overlap, meaning that an organization may be situated inside different "colored" communities, for different "colors," i.e., with regards to different cultural attributes or attitudes that actors possess. In other words, although community constituencies (in organizations) may change across "colors," one can keep account for these changes in order to be able to keep track of the observed recombinations of the community categorizations, when culture enters the game. Obviously, as "colors" change, if two organizations tend to be inregistered in the same "colored" communities more frequently than two other organizations, then one may postulate that the former pair of organizations is more tightly-knit than the latter pair, because their linkage (of belonging to the same community) tends to persist sustaining even under different "colors" (attributes or attitudes). In this way, one may talk of organizational meta-communities, which encapsulate the effect of culture on structure in the observed culturally-driven mutations of the interorganizational network structures. Actually, these culturally constituted meta-communities represent the existing interorganizational alliances, boundaries or cleavages, better than what could have been observed solely in the culture-free interorganizational structure.

Formally, to get the combined cultural-structural meta-community categorization, all we have to do is to form the matrix $M=M(G, C)$ (where $C=\left\{\mathbf{C}^{\alpha_{1}}, \mathbf{C}^{\alpha_{2}}\right.$, $\left.\ldots, \mathbf{C}^{\alpha_{q}}\right\}$ ) with $g$ rows (the surveyed organizations) and $q$ columns (the vector of community partitions with regards all the surveyed cultural attributes or actors' attitudes), the $(i, j)$ entry of which is the index of the community to which the $i$-th organization $O_{i}$ belongs in the community partition $\mathbf{C}^{\alpha_{j}}$ with regards to the $j$-th attribute or attitude $\alpha_{j}$. Of course, $M$ is not a binary matrix, but it can be converted to a binary matrix (still denoted as $M$ ), if we recode its columns by expanding to the corresponding community structure $\left\{C_{1}^{\alpha_{l}}, C_{2}^{\alpha_{l}}, \ldots, C_{c(l)}^{\alpha_{l}}\right\}$, for each cultural attribute $a_{l}$, for $l=1, \ldots, q$. Then, the binary matrix $M$ becomes of order $g \times \sum_{l=1}^{q} c(l)$. Apparently, $M$ represents a two-mode network (a bipartite graph), denoted as $\mathcal{M}$, among organizations and culturally-"colored" communities. 
The corresponding projection of $M$ on just organizations would be an one-mode interorganizational network $\mathcal{N}$ with adjacency matrix equal to the matrix product $M M^{T}$ (i.e., a weighted graph). We call $\mathcal{N}$ "community multiplexity counting interorganizational network," because the $(i, j)$ entry of its adjacency matrix equals to the number of different culturally-"colored" communities, to which the two organizations $O_{i}$ and $O_{j}$ belong. Thus, detecting the community partition of the latter network $\mathcal{N}$, one may determine the structure of meta-communities $\mathfrak{C}=\mathfrak{C}(\mathcal{G})=\left\{\mathcal{C}_{1}, \mathcal{C}_{2}, \ldots, \mathcal{C}_{\mu}\right\}$.

In the next sections, we are going to give two examples of the previous formal methodology and the corresponding computations. These examples intend to manifest the added value of incorporating cultural attributes and individual actors' attitudes in the study of organizational networks. The first example is an artificial one and it concerns a situation, in which there might be no visible community structure in an interorganizational network. However, communities may emerge only as far as one starts "coloring" the patterns of the observed multi-organizational overlaps by considering the cultural attributes that participants may exhibit. The second example is derived from certain concrete empirical data (the IPPS survey, a typical survey about participation in a recent global mobilization) and what it manifests is the fact that incorporating activists' attitudes towards issues of peace and war (together with their membership overlaps) might enhance the evidence about the emergence of a clinging interorganizational alignment (what we call here "meta-communities") among participating social movement organizations, with which surveyed activists are affiliated, due to the existing patterns of shared common attitudes with regards to the salient issues that frame the mobilization, in which activists are participating.

\section{An Artificial Example}

First, let us consider the interorganizational network among four organizations, $A$, $B, C$ and $D$, which is represented in the following weighted graph:

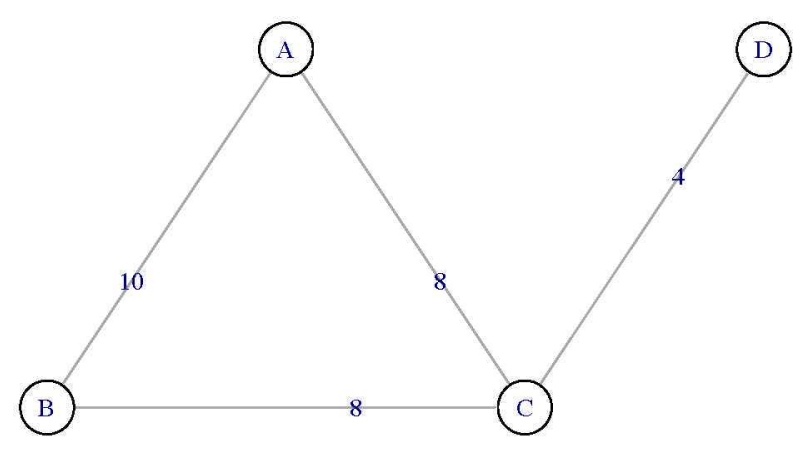

FIGURE 1. A "colorless" (i.e., attribute-free) interorganizational network. 
Here, one can prove (formally) that, for any partition of the "colorless" graph, there exists no positive modularity function, which is maximized over that partition. This means, technically speaking, that the "colorless" interorganizational network of the example does not possess any community structure (in the formal way it was defined previously).

Furthermore, let us assume that participants to the above four organizations exhibit three attributes, denoted by the three "colors," red, blue and green. In particular, let us suppose that the patterns of participants' possession of these four attributes are such that three "colored" interorganizational networks are formed. The "red" interorganizational network is represented in the following weighted graph:

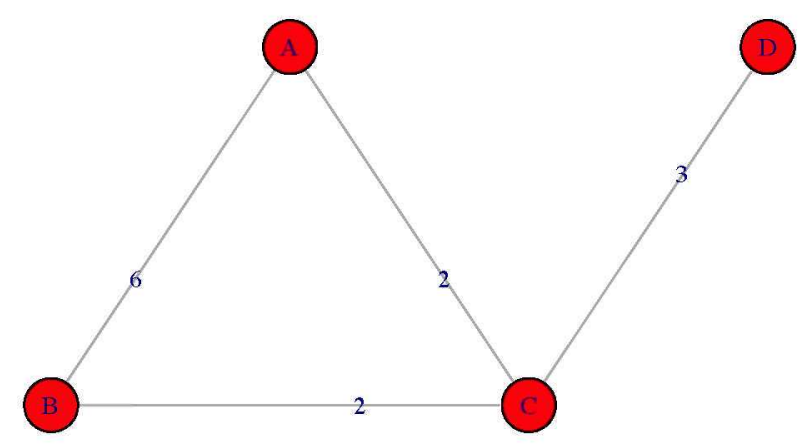

FIGURE 2. The "red" interorganizational network.

Now, it is not difficult to compute that the modularity maximizing community partition of the "red" network admits two communities of organizations: $\{A, B\}$ and $\{C, D\}$.

Next, the "blue" interorganizational network is represented in the following weighted graph:

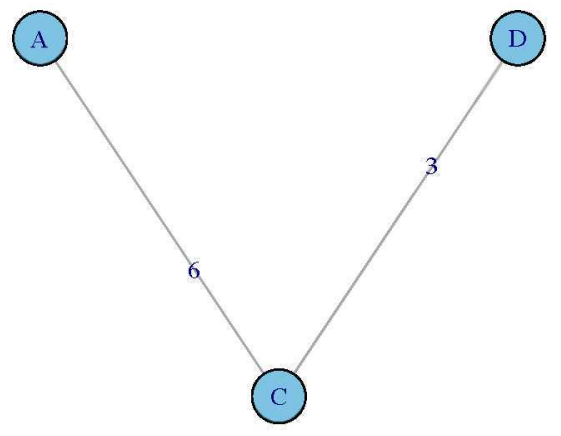

FIGURE 3. The "blue" interorganizational network. 
Again, it is trivial to compute that the modularity maximizing community partition of the "blue" network admits two communities of organizations: $\{A, C, D\}$ and $\{B\}$.

Finally, the "green" interorganizational network is represented by the following weighted graph:

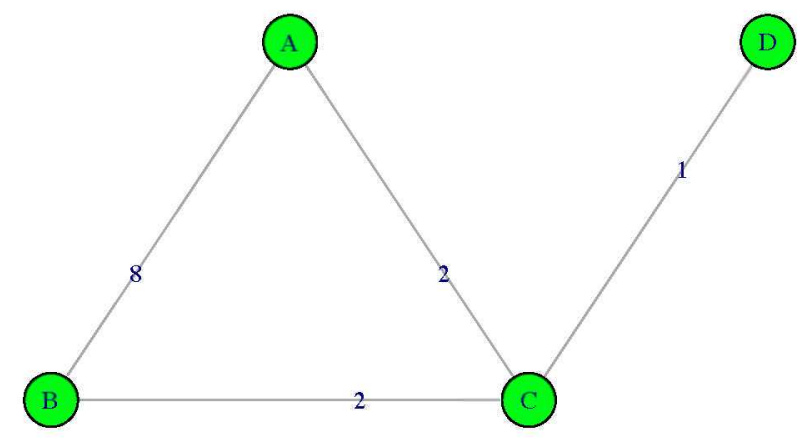

FIGURE 4. The "green" interorganizational network.

Here too, one can easily compute that the modularity maximizing community partition of the "green" network admits three communities of organizations: $\{A, B\},\{C\}$ and $\{D\}$. (Note that, in the last two "colored" partitions, some communities are singletons, i.e., they are composed of single organizations.)

We should admit that, in the previous community detection computations, we have not been taking into consideration the presence of self-loops of nodes. Of course, this is just a technical omission that could be definitely surmounted in a more comprehensive and exhaustive computation, in which one would have to start from the full two-mode data of all participants affiliated to organizations and exhibiting a number of attributes. However, we have made this omission just in order to be able to illustrate the peculiar situation of a community-free "colorless" network coupled with a number of community-available "colored" networks.

Concisely, the distribution of the four organizations in the corresponding "colored" communities that we have found is given by the following table (where cell entries denote the indices of the corresponding communities in the columns):

\begin{tabular}{|c|c|c|c|c|}
\hline \multirow{2}{*}{ Organizations } & \multicolumn{4}{|c|}{ Communities } \\
\cline { 2 - 5 } & "Colorless" & "Red" & "Blue" & "Green" \\
\hline A & - & 1 & 1 & 1 \\
\hline B & - & 1 & 2 & 1 \\
\hline C & - & 2 & 1 & 2 \\
\hline D & - & 2 & 1 & 3 \\
\hline
\end{tabular}

From the above table, one can easily derive what, in the previous section, was called "community multiplexity counting interorganizational network" $\mathcal{N}$ and find that the adjacency matrix of $\mathcal{N}$ is the following: 


$$
\left[\begin{array}{llll}
0 & 2 & 1 & 1 \\
2 & 0 & 0 & 0 \\
1 & 0 & 0 & 2 \\
1 & 0 & 2 & 0
\end{array}\right]
$$

Therefore, in the interorganizational network of this example, two meta-communities emerge, $\{A, B\}$ and $\{C, D\}$, which coincide with the "red" communities, although in the overall network (containing all "colors") no communities could be discerned. Therefore, in certain structurally ambiguous situations that one may encounter in the context of concrete empirical cases, when there appears no community structure to be disclosed by mere structural considerations, then it is substantial that one might focus on any available "colored" cultural attributes in order to be able to discern (at least) some elements of a (possibly partial) community categorization, something which would have been lost in the absence of any focalization on culture.

\section{The Case of Italy in the IPPS Survey}

Let us now come to our second example. This is an interorganizational network, which was extracted from the empirical data (two-mode networks of organizational overlaps in participation and attitudinal possession) of the IPPS survey. In fact, on February 15, 2003, mass protests against the imminent (that period) war in Iraq took place throughout the world, in which more than seven million people in more than 300 cities all over the globe had participated. This globally sustained mobilization was one of the largest peace protests since the Vietnam War on one single day. ${ }^{1}$ On this occasion, an international team of social movement scholars set up a project, called International Peace Protest Survey or IPPS in abbreviation (2003-4), which was coordinated by Stefaan Walgrave of the University of Antwerp in Belgium (Walgrave \& Verhulst, 2009, Diani, 2009). ${ }^{2}$ The aim of this project was to study the demonstrations of the global protest event of February 15, 2003, and compare its social movement dynamics in 8 countries: UK, Italy, the Netherlands, Switzerland, USA, Spain, Germany and Belgium. For this purpose, over 10,000 questionnaires were totally distributed and about 6,000 completed questionnaires were sent back, which makes the successful response rate quite satisfactory (well above $50 \%$ ).

In particular, in the IPPS survey, participating activists (with varying numbers in each country) were asked to declare their affiliation or involvement with the following 16 (types of) organizations:
(1) Church
(5) Political Party
(2) Anti-Racist
(6) Women
(3) Student
(7) Sports - Recreation
(4) Labor Union - Professional
(8) Environmental

\footnotetext{
${ }^{1}$ For more descriptive information see the Wikipedia article http://en.wikipedia.org/wiki/ February_15,_2003_anti-war_protest.

${ }^{2}$ The survey data bases are accessible at http://webh01.ua.ac.be/m2p/index.php?page= project \&\&age $2=$ pproject\&id $=11$.
} 
(9) Art, Music \& Education

(10) Neighborhood

(11) Charitable

(12) Anti-Globalist
(13) Third World

(14) Human Rights

(15) Peace

(16) Other

Furthermore, respondents were asked to answer a number of questions, in which they would express their own opinions and feelings about the meaning, the significance, the reasons, the implications etc. of the war. In particular, activists participating in this event were asked to disclose their positions on the following 10 war-related attitudes:

(1) USA Crusade against Islam

(2) Anti-Dictatorial Regime War

(3) UN Security Council Authorized War

(4) War for Oil

(5) Racist War

(6) Iraqi Threat to World Peace
(7) Always Wrong War

(8) War to Overthrow the Iraqi Regime

(9) Feelings against Neoliberal Globalization

(10) Governmental Dissatisfaction

Here, we are going to discuss just the findings of the analysis of the IPPS survey data from a single country, Italy, where 972 activists, participating in the protest events of February 15, 2003, have responded to the questionnaire. In the following table, we may see what we have found (following the previously discussed analysis) on the distribution of the 16 organizations in various communities formed by the possession of the 10 war-related attitudes by the participating activists. Again, cell entries denote the indices of the corresponding communities in the columns. However, now, the first column corresponds to the case of the "colorless" interorganizational network, in which activists" attitudes were completely ignored, while the last column corresponds to the case of the entirely "colorful" interorganizational network, in which all activists' attitudes were taken into account (incorporated into the community multiplexity counting interorganizational network $\mathcal{N}$, the metacommunity structure of which is given in the last column). 


\begin{tabular}{|c|c|c|c|c|c|c|c|c|c|c|c|c|}
\hline & $\begin{array}{l}\text { No } \\
\text { atti- } \\
\text { tudes }\end{array}$ & $\begin{array}{l}\text { USA } \\
\text { Cru- } \\
\text { sade } \\
\text { again- } \\
\text { st } \\
\text { Is- } \\
\text { lam }\end{array}$ & $\begin{array}{l}\text { Anti- } \\
\text { Dicta- } \\
\text { torial } \\
\text { Regi- } \\
\text { me } \\
\text { War }\end{array}$ & $\begin{array}{l}\text { UN } \\
\text { Se- } \\
\text { cu- } \\
\text { rity } \\
\text { Coun- } \\
\text { cil } \\
\text { Au- } \\
\text { tho- } \\
\text { rized } \\
\text { War }\end{array}$ & $\begin{array}{l}\text { War } \\
\text { for } \\
\text { Oil }\end{array}$ & $\begin{array}{l}\text { Rac- } \\
\text { ist } \\
\text { War }\end{array}$ & $\begin{array}{l}\text { Iraqi } \\
\text { Thr- } \\
\text { eat } \\
\text { to } \\
\text { World } \\
\text { Peace }\end{array}$ & $\begin{array}{l}\text { Al- } \\
\text { ways } \\
\text { Wro- } \\
\text { ng } \\
\text { War }\end{array}$ & $\begin{array}{l}\text { War } \\
\text { to } \\
\text { Over- } \\
\text { throw } \\
\text { the } \\
\text { Iraqi } \\
\text { Reg- } \\
\text { ime }\end{array}$ & $\begin{array}{l}\text { Feel- } \\
\text { ings } \\
\text { again- } \\
\text { st } \\
\text { Ne- } \\
\text { olib- } \\
\text { eral } \\
\text { Glob- } \\
\text { al- } \\
\text { iza- } \\
\text { tion }\end{array}$ & $\begin{array}{l}\text { Gov- } \\
\text { ern- } \\
\text { ment- } \\
\text { al } \\
\text { Dis- } \\
\text { sat- } \\
\text { is- } \\
\text { fac- } \\
\text { tion }\end{array}$ & $\begin{array}{l}\text { All } \\
\text { atti- } \\
\text { tudes }\end{array}$ \\
\hline Church & 8 & 7 & 4 & 1 & 8 & 6 & 5 & 3 & 1 & 5 & 3 & 2 \\
\hline Anti-Racist & 3 & 6 & 1 & 4 & 2 & 2 & 2 & 7 & 2 & 1 & 3 & 3 \\
\hline Student & 4 & 6 & 3 & 3 & 3 & 4 & 5 & 1 & 1 & 1 & 4 & 2 \\
\hline Labor Union - Prof. & 1 & 4 & 2 & 3 & 1 & 7 & 1 & 6 & 6 & 4 & 3 & 1 \\
\hline Political Party & 1 & 4 & 2 & 3 & 1 & 7 & 1 & 6 & 6 & 4 & 4 & 1 \\
\hline Women & 5 & 1 & 1 & 2 & 4 & 5 & 2 & 8 & 2 & 1 & 3 & 3 \\
\hline Sport - Recr. & 7 & 5 & 3 & 2 & 6 & 6 & 5 & 4 & 1 & 2 & 2 & 2 \\
\hline Environmental & 2 & 6 & 1 & 4 & 5 & 2 & 6 & 9 & 2 & 1 & 3 & 3 \\
\hline Art, Music \& Edu. & 7 & 5 & 3 & 2 & 6 & 6 & 5 & 4 & 1 & 2 & 1 & 2 \\
\hline Neighborhood & 9 & 3 & 3 & 3 & 9 & 1 & 7 & 2 & 6 & 3 & 3 & 1 \\
\hline Charitable & 6 & 2 & 1 & 1 & 7 & 6 & 3 & 5 & 3 & 6 & 1 & 2 \\
\hline Anti-Globalist & 3 & 8 & 1 & 4 & 2 & 3 & 4 & 7 & 4 & 1 & 3 & 3 \\
\hline Third World & 3 & 8 & 1 & 4 & 2 & 3 & 4 & 7 & 4 & 1 & 1 & 3 \\
\hline Human Rights & 3 & 8 & 1 & 4 & 2 & 3 & 4 & 7 & 4 & 1 & 1 & 3 \\
\hline Peace & 3 & 8 & 1 & 4 & 2 & 3 & 4 & 7 & 4 & 1 & 4 & 3 \\
\hline Other & 6 & 2 & 1 & 1 & 7 & 5 & 3 & 5 & 5 & 5 & 4 & 2 \\
\hline
\end{tabular}

The following two graphs portray the interorganizational network of activists, who have participated in the February 15, 2003, protest events in Italy (as they were surveyed by IPPS). In these graphs, the participating organizations are colored according to the community or meta-community they belong: on the first graph, when activists' attitudes are ignored in the interorganizational network $\mathcal{G}$, and, on the second graph, when all these attitudes are cumulated in the community multiplexity counting interorganizational network $\mathcal{N}$.

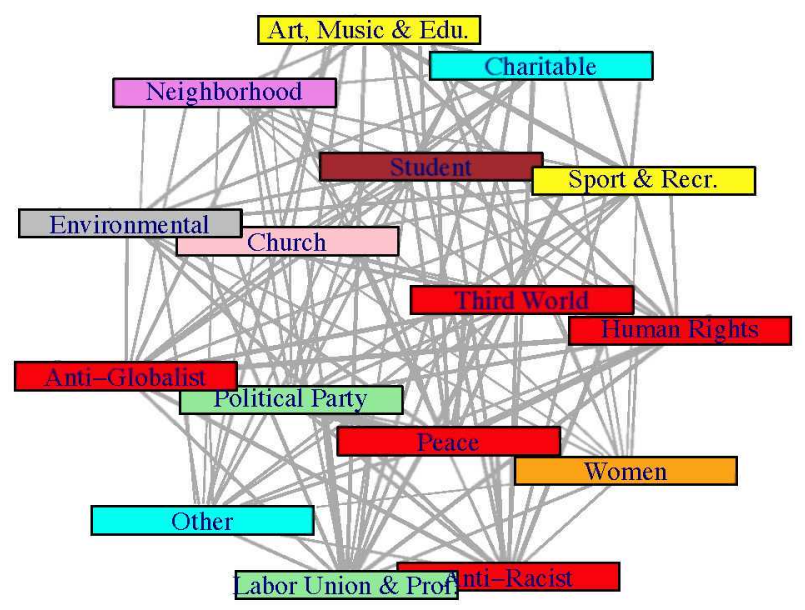

FiguRE 5. The interorganizational network $\mathcal{G}$ in Italy ignoring activists' attitudes and portraying the corresponding communities of the participating organizations with different colors. 


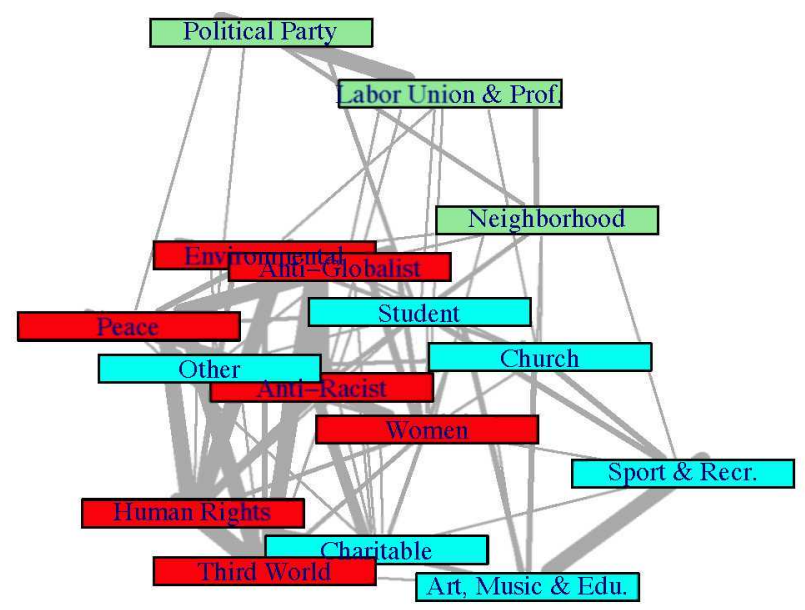

FigURE 6. The community multiplexity counting interorganizational network $\mathcal{N}$ in Italy taking into account all activists' attitudes and portraying the corresponding meta-communities of the participating organizations with different colors.

The comparison between communities (or meta-communities) of organizations in these two networks is fascinating. First of all, the "colored" community categorization (i.e., taking into account activists' attitudes) is more succinct - composed of 4 meta-communities - than the "colorless" one (in the absence of activists' attitudes) - composed of 9 communities. To start with, from the above two interorganizational graphs, it is evident that there exist two attitudinally derived meta-communities that almost coincide with two corresponding structural communities. First, we have a group of five organizations (Anti-Globalist, Peace, Anti-Racist, Human Rights and Third World), which stick together into the same meta-community (and, very frequently, they appear into the same "colored" communities too), but they also constitute one of the 9 communities of the attitudes-free interorganizational structure. Note that the full constituency of this meta-community (in Italy) includes two more organizations, Environmental and Women, which were structurally classified in communities composed of single organizations (singletons). Incidentally, based on purely structural considerations, Mario Diani (2009) has classified the meta-cluster of these seven organizations as the group of "peace protest communities." Secondly, there exists a group of two organizations, Political Party and Labor Union - Professional, which, just by themselves, compose a single structural community, but in the inter-attitudinal network they partake with a third organization, Neighborhood, in the constitution of one of the existing three meta-communities. In these two cases, one could only speculate that the effect of the attitudinally expressed culture into the emergence of the structural concrescence of the constituent organizations is so profound that it cannot be singled out from the purely structural interorganizational entanglement of the observed overlapping memberships.

Finally, coming back to the Italian protest network, there are cases manifesting how cultural influence (through the possession of war-related attitudes by 
protesters) may facilitate the emergence of some coherent interorganizational metacommunities, which in the "colorless" network were fragmented and dispersed in disparate structural communities. A very characteristic example is the appearance of a meta-community composed of five organizations (in Italy), resembling what in the terminology of Mario Diani (2009) was called "peace associations": Student, Church, Sports-Recreation, Charitable and Art, Music \& Education (a sixth "organization" in this meta-community is the residue "Other"). In the structural community categorization, only two of these six organizations were clustered in the same community (Sports-Recreation and Art, Music \& Education), while the remaining three were structurally classified in communities composed of singletons.

We conclude this section by showing the total meta-community multiplexity counting network, which is formed by gluing together the outcomes of the analyses of all the eight countries partaking in the IPPS survey (similar to the Italian case that we have presented above).

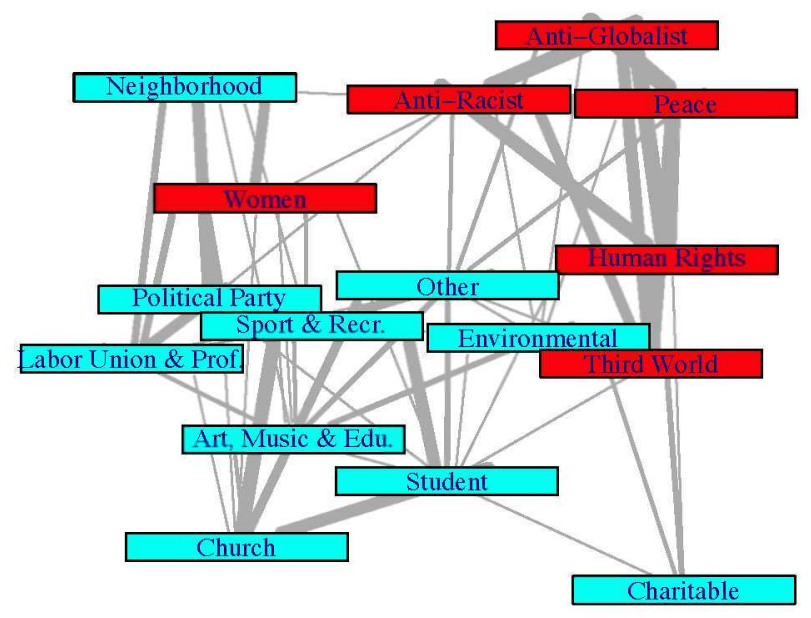

FIGURE 7. The meta-community multiplexity counting interorganizational network in the eight countries of the IPPS survey, taking into account the attitudes of all activists' (from all countries) and portraying the corresponding meta-communities of the participating organizations with different colors.

Now, the emerging meta-communities at the international level are just two. One is the meta-community of the six organizations: Anti-Globalist, Peace, AntiRacist, Human Rights, Third World and Women. The other is the meta-community of the remaining nine organizations (exempting the residual Others): Environmental, Student, Church, Sports-Recreation, Charitable, Art, Music \& Education, Political Party, Labor Union \& Professional and Neighborhood. This interorganizational cleavage is daringly reminiscent of Mario Diani's (2009) contradistinction between "protest communities" and "associations." However, the difference is that now the cleavage has been derived analytically (by a formal methodology on a combined structural-cultural community categorization) than been speculated a priori 
in the context of a plain structural analysis, which would have discarded any available cultural-attitudinal evidence in the survey.

\section{Discussion}

Let us start by commenting on a seemingly rather convincing negative assessment of the utility of cultural availability and significance to studies of social movement participation that has been made a long time ago by Doug McAdam (1986) and it seems that it may have discouraged the attention of many social movement scholars on a possible investigation of the structure-culture duality in the context of participation studies. This is the argument of the "attitudinal affinity" that McAdam exposes very clearly when he argues (p. 65): "The argument is that structural availability is more important than attitudinal affinity in accounting for differential involvement in movement activity. Ideological disposition toward participation matters little if the individual lacks the structural contact to 'pull' him or her into protest activity." McAdam is right, as many recent empirical studies have been demonstrating, that the decisive and sometimes even predictive role in encouraging activism or recruitment to at least high-risk forms of social movement participation is rather structural than cultural or attitudinal. However, when it comes to explaining the emergence of concrete structural patterns of certain macro-level interorganizational features (as, for example, the diversity in the observed alignments in particular community categorizations), the structural argument is completely mute. It might be true that an individual is not pulled into protest activity because of ideological dispositions or cultural-attitudinal affinities, but because of the structural interlocking within certain constricting multi-organizational membership overlaps. Nevertheless, a mere count of constituencies of structural interorganizational interlockings does not suffice to justify why and to account how such alignments or other particularized patterns come to exist.

Yes, it is indeed reasonable to accept the structural rationale that two organizations, say, $X$ and $Y$, should be inter-connected as far as they do accommodate some considerable number of overlapping members. Moreover, under the structural rationale, we can draw a map (a graph of the corresponding interorganizational network) portraying the entanglements among the relational positions of all observed organizations. But this graph is typically highly dense (because of the often high rates of observed co-membership overlaps) and it might be the case that the two organizations $X$ and $Y$ were still connected in it through a moderate rate of co-memberships. However, if it also turns out that $X$ and $Y$ are situated within different parts of this interorganizational network (for instance, they may be aligned

within the camps of two different structural communities) or they might exhibit some diverse structural features (like centralities, core-periphery location, equivalences etc.), then how can the purely structural argument explain or construe such circumstances? No, it can't. This is why the structurally unwanted patterns of cultural or attitudinal affinities need to be called back in order to elucidate such structural differentiations by inspecting their nuances with a palette of "colors" (i.e., cultural distinctions or significations), which might revive the existing diversity that the structural rationale has cached away. 
To give a simpler example, say, the overlapping members between two pairs of organizations are 50 in both cases. But this is just a number and the structural rationale is basing all its explanatory power on the number 50 in relation to other such numbers counting the existing membership overlaps among all other pairs of organizations. Nevertheless, one does not need any sophisticated philosophical argument to understand that, in reality, there are qualities hidden beyond the surface of quantities, which are counting various entities in the world. It is sufficient to argue that the 50 participants interlocking one pair of organizations may be either (i) the same or (ii) completely different or (iii) overlapping with the 50 participants interlocking another pair of organizations. Of course, it is not a matter of a strict identification of the names of participants, since most of social movement surveys are necessarily anonymous (or they have to be anonymized, when their findings are disseminated in public). However, participants possess qualities and often they are willing to disclose them through the responses they give in social surveys. This is the case of the cultural or attitudinal characteristics that participants might be asked in a survey to expose. Then, in the light of certain explicitly expressible cultural diversities, the two quantitative interlocks of a mere count of 50 co-participants might be decomposed into two different arrays of participants endowed with different cultural or attitudinal characteristics. Actually, this is the behavioral evidence needed to explain the emergence of macro-patterns, like community categorizations or alignments or boundaries among different positional locations, in a structurally composed interorganizational network.

That's why culture matters in studies of structure. To grasp the cultural significance to structure in full-fledged terms presumes that one has to surpass an old methodological bias about the marginal role that individual actors' attitudes might play in structural analyses. However, it was from the 1980 s that Bonnie Erickson has been shouting loudly that the right structural way to comprehend how attitudes really work is by considering that: "(a) natural units of analysis for attitudes are not isolated individuals but social networks and (b) viable subjects for explanation are not individual attitudes, but degrees of attitude agreement among individuals in given structural situations" (Erickson, 1988, p. 99). This is exactly what the proposed formal methodology of the culture-structure duality is aspiring to do. It is not a matter of aggregating individual actors' attitudes in a structural group that they may form - one knows it very well, if one understands the problem with the ecological fallacy. On the contrary, it is a matter of forming the inter-attitudinal network by aggregating in community categorizations the cultural patterns of "degrees of attitude agreement among individuals" and, subsequently, juxtaposing them with the structural pattern of membership overlaps and interlocks in the interorganizational network. This is exactly the approach we have been following here.

Now, coming back to a discussion of some more formal aspects of the proposed methodology of an interorganizational network analysis based on the structureculture duality, let us first remark on something that one might have already noticed in the previous IPPS example. The cardinality of the culturally-driven meta-communities appeared there to be much lower than the cardinality of the purely structural communities. However, this is completely consistent with the fact 
that the number of links in the interorganizational network $\mathcal{G}$ (i.e., the number of overlapping memberships of participants in organizations) is much higher than the number of links in the community multiplexity counting network $\mathcal{N}$ (i.e., the number of overlapping memberships of organizations in organizational communities, which can be at most as many as the number of the reported attitudes, i.e., 10 in that example). Unless, of course, one was analyzing empirical data containing a very large number of cultural attributes or actors' attitudes and a relatively smaller number of organizations (or events etc.), to which actors could have been interlocked. This was not the case with the IPPS data: a total number of a few thousands of activists (in eight countries) generating a very large number of tightknit overlapping members of totally 16 organizations, while the surveyed attitudes were just 10. But, as said, there might be empirical data, in which the numbers of organizational affiliations could be much smaller than the numbers of attributional (or attitudinal) affiliations. Perhaps, in such a case, it would be advisable to reverse the order of analysis: one could first start with the inter-cultural structure (forming the inter-attributional or inter-attitudinal network) and, subsequently, one could proceed in decomposing the latter structure according to the sparser patterns of the organizational affiliations. In fact, this reversed analysis would signify a good grip on the (reversible) duality between structure and culture.

Furthermore, from the examples and formal network discussion that we have made, it is clear that, in the context of networks of participation, culture, through the incorporation of attitudes that activists happen to possess, may play a cementation role in forcing certain organizations to align inside the same meta-communities, while these organizations, in the absence of cultural attitudes, would appear inexplicably clustering within certain dissimilar structural groups. As a matter of fact, global cultural identities in modern societies may still play the role of a functional substitute for local communities, as, for instance, nation and nationalism once used to provide the integrative cement that made modern communities appear as the Gemeinschaft (Haas, 1964, p. 465). Actually, in the contemporary world, this is exactly what one may observe analytically in social networks and, in particular, with regards to the constricting effect of culture on structure, as it is typically celebrated by the notion of the "global village," attributed to Marshall McLuhan.

However, the indication that a cultural-attitudinal analysis may sometimes return the same organizational clusters with the ones obtained by a pure structural analysis should not be misleading. By all means, this does not mean that the cultural-attitudinal analysis is redundant. In many other cases (as we have already seen in the Italian IPPS network), the cultural-attitudinal analysis may shed light on circumstances that would have remained impalpable from the structural point of view. The problem with the structural analysis of interorganizational networks is that, as a network theory, it is extremely coarse (for instance, because of the oversimplification caused by the reduction-projection of a two-mode network on an one-mode network, which dispenses with any existing micro-structural information). Therefore, the interorganizational structural analysis remains strictly descriptive in character and it is incapable of providing the required background of (say) some precise micro-localities (like local independences or other complex or fractal topological features), over which one could apply certain powerful recently 
developed statistical or computational techniques (like ERGMs, multi-level analyses, small-worlds, power laws etc.) in order to be able to estimate or simulate the outcome of those random processes or micro-social mechanisms that could predict more accurately (and formally rigorously) the routes of the dynamic evolution of structure.

Of course, neither the cultural-attitudinal analysis may do this. However, the added value of the latter to a plain, if not exiguous, form of structural analysis is that culture may provide an empirical heuristic for the interpretation of structure, which would have otherwise remained insubstantially "colorless" or insipid. Such is the lesson of some macro-sociological or practice-oriented institutional approaches built over the fundamental comprehensive idea of the structure-culture duality (Breiger, 2000, Breiger \& Mohr, 2004, Lizardo, 2006). It is essential that social network analysts become more acclimated to this lesson, because they still have many contributions to offer from the angle of the methodological clarity and the rigorous formalism of the network-relational paradigm.

\section{REFERENCES}

Andretta, Massimiliano, Botetzagias, Iosif, Boudourides, Moses, Kioufegi, Olga, \& Yang, Mundo. 2009. Novel Characteristics of the GJMs: A (latent) network approach. Pages 149-172 of: della Porta, Donatella (ed), Another Europe: Conceptions and Practices of Democracy in the European Social Forums. London: Routledge. http://nicomedia.math.upatras.gr/sm/Demos2009.pdf.

Brandes, Ulrik, Delling, Daniel, Gaertler, Marco, Görke, Robert, Hoefer, Martin, Nikoloski, Zoran, \& Wagner, Dorothea. 2008. On modularity clustering. IEEE Transactions on Knowledge and Data Engineering, 20, 172-188. http://dx. doi.org/10.1109/TKDE.2007.190689.

Breiger, Ronald L. 1974. The duality of persons and groups. Social Forces, 53, 181-190. http://www. jstor.org/stable/2576011.

Breiger, Ronald L. 2000. A tool kit for practice theory. Poetics, 27, 91-115. http://dx.doi.org/10.1016/S0304-422X(99)00026-1.

Breiger, Ronald L., \& Mohr, John W. 2004. Institutional logics from the aggregation of organizational networks: Operational procedures for the analysis of counted data. Computational \& Mathematical Organization Theory, 10, 17-43. http: //dx.doi.org/10.1023/B: CMOT.0000032578.16511.9d.

Calhoun, Craig J. 2009. Community: Toward a variable conceptualization for comparative research. Social History, 5, 105-129. http://www. jstor.org/pss/ 4284951.

Castoriadis, Cornelius. 1998. The Imaginary Institution of Society. Transl. Kathleen Blamey. Cambridge: MIT Press.

Cornwell, Benjamin, \& Harrison, Jill Ann. 2004. Union members and voluntary associations: Membership overlap as a case of organizational embeddedness. American Sociological Review, 69, 862-881. http://dx.doi.org/10.1177/ 000312240406900606.

Diani, Mario. 2004. Networks and Participation. Pages 339-359 of: Snow, David A, Soule, Sarah A., \& Kriesi, Hanspeter (eds), The Blackwell Companion to Social Movements. Oxford: Blackwell. 
Diani, Mario. 2009. The structural bases of protest events: Multiple memberships and civil society networks in the 15 February 2003 anti-war demonstrations. Acta Sociologica, 52, 63-83. http://dx.doi.org/10.1177/0001699308100634.

Emirbayer, Mustafa. 1997. Manifesto for a Relational Sociology. American Journal of Sociology, 103(2), 281-317. http://dx.doi.org/10.1086/231209.

Emirbayer, Mustafa, \& Goodwin, Jeff. 1994. Network analysis, culture, and the problem of agency. American Journal of Sociology, 99, 1411-1454. http://www . jstor.org/stable/2782580.

Emirbayer, Mustafa, \& Mische, Ann. 1998. What is agency? American Journal of Sociology, 103, 962-1023. http://dx.doi.org/10.1086/231294.

Erickson, Bonnie H. 1988. The relational basis of attitudes. Pages 99-121 of: Wellman, Barry, \& Berkowitz, S. D. (eds), Social Structures: A Network Approach. Cambridge: Cambridge University Press.

Fararo, Thomas J., \& Doreian, Patrick. 1984. Tripartite structural analysis: Generalizing the Breiger-Wilson formalism. Social Networks, 6, 141-175. http: //dx.doi.org/10.1016/0378-8733(84)90015-7.

Feld, Scott L. 2000. The focused organization of social ties. American Journal of Sociology, 86, 1015-1035. http://www. jstor.org/stable/2778746.

Fortunato, S. 2010. Community detection in graphs. Phys. Rep., 486, 75-174. http://dx.doi.org/j.physrep.2009.11.002.

Frank, Kenneth A. 1995. Identifying cohesive subgroups. Social Networks, 17, 27-56. http://dx.doi.org/10.1016/0378-8733(94)00247-8.

Friedland, Roger, \& Mohr, John. 2004. The cultural turn in American sociology. Pages 1-10 of: Friedland, Roger, \& Mohr, John (eds), Matters of Culture: Cultural Sociology in Practice. Cambridge: Cambridge University Press.

Gulati, Ranjay, \& Gargiulo, Martin. 1999. Where do interorganizational networks come from? American Journal of Sociology, 104, 1439-1493. http://www. jstor.org/stable/2990941.

Haas, Ernst B. 1964. Beyond the Nation-State: Functionalism and International Organization. Stanford: Stanford University Press.

Hedström, Peter, Sandell, Rickard, \& Stern, Charlotta. 2000. Mesolevel networks and the diffusion of social movements: The case of the Swedish Social Democratic Party. American Journal of Sociology, 106, 145-172. http://www . jstor.org/ pss/10.1086/303109.

Lamont, Michèlle, \& Molnár, Virág. 2002. The study of boundaries in the social sciences, Annual Review of Sociology, 28, 167-195. http://dx.doi.org/10. 1146/annurev. soc.28.110601.141107.

Lazarsfeld, Paul F., \& Merton, Robert K. 1964. Friendship as social process: A substantive and methodological analysis. Pages 18-66 of: Berger, Monroe, Abel, T., \& Page, C. H. (eds), Freedom and Control in Modern Society. New York: Octagon Books.

Leenders, Roger Th. A. J. 1997. Longitudinal behavior of network structure and actor attributes: Modeling interdependence of contagion and selection. Pages 165-184 of: Doreian, Patrick, \& Stokman, Franz N. (eds), Evolution of Social Networks. Amsterdam: Gordon \& Breachs.

Lizardo, Omar. 2006. How cultural tastes shape personal networks. American Sociological Review, 71, 778-807. http://dx.doi.org/10.1177/ 000312240607100504. 
McAdam, Doug. 1986. Recruitment to high-risk activism: The case of freedom Summer. American Journal of Sociology, 92, 64-90. http://www.jstor.org/ stable/2779717.

McPherson, Miller, Smith-Lovin, Lynn, \& Cook, James M. 2001. Birds of a feather: Homophily in Social Networks. Annual Review of Sociology, 27, 415-444. http: $/ / \mathrm{dx}$.doi.org/10.1146/annurev.soc.27.1.415.

Melucci, Alberto. 1989. Nomads of the Present. Philadelphia: Temple University Press.

Melucci, Alberto. 1996. Challenging Codes. Cambridge: Cambridge University Press.

Moody, James, \& White, Douglas R. 2003. Structural cohesion and embeddedness: A hierarchical concept of social groups. American Sociological Review, 68, 103127. http://dx.doi.org/10.1234/12345678.

Mützel, Sophie. 2009. Networks as culturally constituted processes. Current Sociology, 57(6), 871-887. http://dx.doi.org/10.1177/0011392109342223.

Newman, Mark E.J., \& Girvan, Michelle. 2004. Finding and evaluating community structure in networks. Phys. Rev. E, 69, 026113. http://dx.doi.org/10.1103/ PhysRevE.69.026113.

Robins, Garry, Pattison, Philippa, \& Elliott, Peter. 2001a. Network models for social influence processes. Psychometrika, 66, 161-190. http://dx.doi.org/ 10.1007/BF02294834.

Robins, Garry, Elliott, Peter, \& Pattison, Philippa. 2001b. Network models for social selection processes. Social Networks, 23, 1-30. http://dx.doi.org/10. 1016/S0378-8733(01)00029-6.

Robins, Garry, Pattison, Philippa, Kalish, Yuval, \& Lusher, Dean. 2007. An introduction to exponential random graph $\left(p^{*}\right)$ models for social networks. Social Networks, 29, 173-191. http://dx.doi.org/10.1016/j.socnet.2006.08.002.

Silver, Allan. 1989. Friendship and trust as moral ideals: An historical approach. European Journal of Sociology, 30, 274-297. http://dx.doi.org/10.1017/ S0003975600005890.

Silver, Allan. 1990. Friendship in Commercial Society: Eighteenth-Century Social Theory and Modern Sociology. American Journal of Sociology, 91, 1474-1504. http://www.jstor.org/stable/2780332.

Simmel, Georg. 1955. Conflict and the Web of Group Affiliations. Transl. Kurt H. Wolff and Reinhard Bendix. New York: Free Press.

Steglich, Christian, Snijders, Tom A.B., \& Pearson, Michael. 2007. Dynamic networks and behavior: Separating selection from influence. Socialogical Methodology, 40, 329-393. http://dx.doi.org/10.1111/j.1467-9531.2010.01225.x.

Walgrave, Stefaan, \& Verhulst, Joris. 2009. Government stance and internal diversity of protest: A comparative study of protest against the war in Iraq in eight countries. Social Forces, 87, 1355-1387. http://dx.doi.org/10.1353/sof.0. 0171.

Wasserman, Stanley, \& Faust, Katherine. 1994. Social Network Analysis: Methods and Applications. 1st edn. Cambridge: Cambridge University Press.

White, Harrison C. 2008a. Identity and Control: How Social Formations Emerge. 2nd edn. Princeton, NJ: Princeton University Press.

White, Harrison C. 2008b. Notes on the constituents of social structure. Soc. Rel. 10 - Spring 1965. Sociologica, 1, 1-15. http://dx.doi.org/10.2383/26576. 
Department of Mathematics, University of Patras, Greece

E-mail address: Moses.Boudourides@gmail.com 